\title{
X-ray Emission Processes in Extragalactic Jets, Lobes and Hot Spots
}

\author{
Andrew S. Wilson \\ Astronomy Department, University of Maryland, College Park, MD 20742
}

\begin{abstract}
This paper is a brief review of the processes responsible for X-ray emission from radio jets, lobes and hot spots. Possible photons in inverse Compton scattering models include the radio synchrotron radiation itself (i.e. synchrotron self-Compton [SSC] emission), the cosmic microwave background (CMB), the galaxy starlight and radiation from the active nucleus. SSC emission has been detected from a number of hot spots. Scattering of the CMB is expected to dominate for jets (and possibly hot spots) undergoing bulk relativistic motion close to the direction towards the observer. Scattering of infrared radiation from the AGN should be observable from radio lobes, especially if they are close to the active nucleus. Synchrotron radiation is detected in some sources, most notably the jet of M87. I briefly discuss why different hot spots emit X-rays by different emission mechanisms and the nature of the synchrotron spectra.
\end{abstract}

Key words: galaxies:active, galaxies:jets, galaxies:nuclei, magnetic fields, X-rays:galaxies

\section{Introduction}

X-ray emission from diffuse cosmic gases may result from thermal or non-thermal processes. An X-ray emitting thermal gas can be collisionally ionized, in which case the temperature needs to be $\simeq 10^{7-8} \mathrm{~K}$, or photoionized, when the temperature is much lower (several $\times 10^{4} \mathrm{~K}$ -

Email address: wilson@astro.umd.edu (Andrew S. Wilson).

Preprint submitted to Elsevier Science several $\left.\times 10^{5} \mathrm{~K}\right)$. Chandra and XMM observations have shown that the latter type of gas is responsible for the X-ray emission of several bright Seyfert galaxies. For example, in NGC 1068 the widths of the radiative recombination continua reveal that the temperature is only a few $x$ $10^{4} \mathrm{~K}$, indicating the gas is photoionized (and photoexcited) with a range of ionization parameters (Kinkhabwala et al. 2002; Brinkman et al. 2002). The non-thermal processes are inverse Compton (IC) scattering and synchrotron radiation. The ener- 
gies of the relativistic electrons tend to be low if the radiation results from IC emission (Section 2) and high if it results from synchrotron radiation $\left(\gamma=\mathrm{E} / \mathrm{mc}^{2} \simeq 10^{7}-10^{8}\right.$ for radiation in the Chandra band and a canonical $\mathrm{B}=10^{-4}$ gauss magnetic field strength).

Thermal X-ray emission has not, to my knowledge, been detected from any extragalactic jet. Detection of IC emission allows the magnetic field strength to be measured directly as long as the relevant photon density is known and there is no bulk relativistic motion. If the $\mathrm{X}$-rays are synchrotron, we infer the presence of high energy electrons and/or positrons with synchrotron half lives or order years, in which case X-ray images highlight regions of current particle acceleration.

Bulk relativistic motion is described by the Doppler parameter $\delta=[L(1-\beta \cos \theta)]^{-1}$, where $L$ is the Lorentz factor of the bulk flow, $\beta$ is the bulk velocity in units of the speed of light, and $\theta$ is the angle between the velocity vector and the line of sight. If $\mathrm{S}_{\nu}^{\prime} \propto \nu^{-\alpha}$ is the flux density which would be observed in the absence of bulk motion and the "blob" emits isotropically in its own frame, the observed spectrum $\mathrm{S}_{\nu} \propto \delta^{3+\alpha} \mathrm{S}_{\nu}^{\prime}$ (e.g. Begelman et al. 1984). This dependence on $\delta$ is usually assumed for synchrotron and synchrotron selfCompton (SSC) emission. On the other hand, if the radiation being scattered is isotropic in the Hubble (or galaxy) frame (so called external Compton [EC] emission), then the principal dependence of the observed spectrum is $\mathrm{S}_{\nu} \propto \delta^{4+2 \alpha} \mathrm{S}_{\nu}^{\prime}$ (Dermer 1995). The magnetic field derived from SSC or EC models is then a function of $\delta$, the function being different in the two cases (e.g. Tavecchio et al. 2000; Celotti et al. 2001).

\section{Inverse Compton Scattering - Sources of Photons}

A key feature of IC models is that the spectral index of the X-ray emission should be the same as that of the synchrotron emission from the same population of electrons. Thus measurement of the X-ray spectral index is vital for confirmation of IC models. However, the synchrotron emission may be at too low a frequency to be observable. Possible sources of photons in IC models include:

a) the synchrotron radiation itself i.e. SSC emission.

b) the cosmic microwave background radiation $(\mathrm{CMB})$. To scatter this radiation into the Chandra band requires $\gamma \simeq 10^{3}$. Such electrons radiate synchrotron radiation at meter wavelengths. As pointed out by Schwartz (2002), the enhancement of the CMB energy density by a factor of $(1+z)^{4}$ compensates for the (1 $+z)^{-4}$ decrease of surface brightness with increasing redshift, $\mathrm{z}$, and X-ray emission from such relativistic jets pointed towards us should be seen to large distances in the universe.

c) the galaxy starlight. Scattering into the Chandra band would require very low energy $(\gamma \simeq 20)$ cosmic 
rays. Such electrons would radiate synchrotron radiation at $\simeq 0.1 \mathrm{MHz}$ for our canonical $\mathrm{B}=10^{-4}$ gauss, too low a frequency to be observable.

d) radiation from the active nucleus, which may be "beamed" along the radio axis. The radiation density is probably dominated by infrared emission, so we require $\gamma \simeq 100-300$, which would radiate synchrotron radiation at a few to tens of $\mathrm{MHz}$.

In the following, I provide examples of each of the different radiation processes.

\section{Inverse Compton Scattering}

\subsection{Synchrotron Self-Compton Emission}

Chandra has detected all four of the radio hot spots (A, B, D and $\mathrm{E}$ ) in Cygnus $\mathrm{A}$ and obtained spectra for the two brightest ones (A and D). Both are well described by a power law with spectral index $\alpha=0.8 \pm$ 0.2 absorbed by the Galactic column in the direction of Cygnus A (Wilson et al. 2000). Thermal X-ray models require too high gas densities and may be ruled out. The images and spectra strongly support SSC models of the X-ray emission, as proposed by Harris et al. (1994) on the basis of ROSAT imaging observations. Such models indicate that the magnetic field in each of the brighter hot spots is $1.5 \times 10^{-4}$ gauss, with an uncertainty of a few tens of percent. This field strength is close to equipar- tition. A few other cases of SSC emission from hot spots are known (e.g. 3C 295 - Harris et al. 2000; 3C 123 Hardcastle et al. 2001) and the number is likely to increase dramatically with further Chandra observations.

In order to check the predictions of the SSC model, P. L. Shopbell and I have observed the hot spots of Cygnus A with HST and found evidence for diffuse optical emission on the northern edge of hot spot D. The apparent displacement of the optical emission from the peak of the radio and X-ray emissions may result from astrometric uncertainties in the HST data. The strength of the optical emission is consistent with, though somewhat higher than, the predictions of the SSC model (Fig. 1). However, this optical emission needs to be confirmed by deeper HST observations.

\subsection{IC Scattering of the CMB}

The quasar PKS0637-752 was the first celestial target of Chandra. The observations revealed an X-ray jet coincident with the inner portion of the jet previously detected in the radio (Schwartz et al. 2000). Schwartz et al. (2000) noted that an SSC model implies extreme departures from the conventional minimum energy criterion. Tavecchio et al. (2000) and Celotti et al. (2001) showed that IC scattering of the CMB is consistent with equipartition for a Doppler factor $\delta \simeq 10$. This process is plausible since VLBI observations reveal superluminal motion on pc scales, 


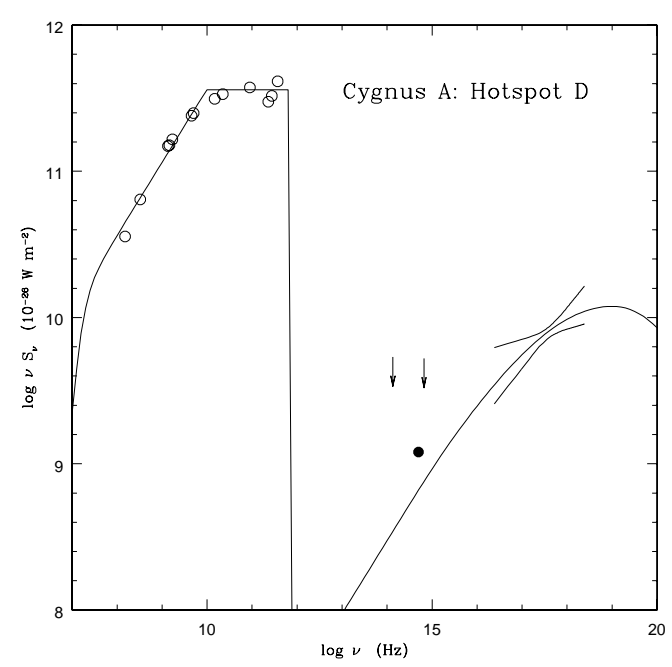

Fig. 1. The spectrum of hot spot D in Cygnus A (adapted from Wilson et al. 2000). The pentagons represent radio measurements, the two downward pointing arrows represents upper limits to the optical and near infrared emissions from ground-based measurements (Meisenheimer et al. 1997), the filled circle represents a tentative detection (requiring confirmation) with HST at $\simeq$ $6000 \AA$ and the bow tie is the Chandra-measured boundary of the X-ray spectrum (90\% confidence). The continuous line is a representation of the radio spectrum and the predicted SSC spectrum for $\mathrm{B}=1.5 \times 10^{-4}$ gauss.

implying $\theta<6^{\circ}$. This interpretation implies that the bulk motion of the jet is relativistic at nuclear distances up to several hundred kpc, a result consistent with systematic polarisation asymmetries in powerful radio galaxies and quasars (Laing 1988; Garrington et al. 1988).

We independently evaluated this process for the X-ray emission of the 100 kpc long jet of Pictor A (Wilson et al. 2001). There it was found that an equipartition model requires $\theta \simeq 8^{\circ}$, which is too small for this lobe dom- inated radio galaxy. For more plausible values of $\theta$, the magnetic field would be below equipartition.

\subsection{IC Scattering of Nuclear Pho- tons}

In the unified model of AGN, the nucleus is surrounded by a disk or torus of gas and dust. This structure collimates much of the radiation into two wide cones along and around the radio axis. Thus the lobes of radio galaxies are expected to be illuminated by intense radiation from a "hidden" quasar. Additionally, there may be a much narrower "blazar" beam emitted by a relativistic jet in the nucleus. Relativistic electrons with $\gamma \sim 100-300$ will IC scatter infrared photons from the quasar into the Chandra band. This process should dominate scattering of the CMB for sources whose axis is not close to the line of sight, for radio lobes/jets with only mild or nonrelativistic bulk motion and for radio lobes/jets close to the nucleus.

Brunetti et al. (1997) have calculated the anisotropic IC scattered emission for this situation. Because of the angle dependence of the IC scattering, the more distant lobe is predicted to be somewhat brighter, depending on the value of $\theta$. Brunetti et al. (2001, 2002) have argued that diffuse X-ray emission from radio lobes in 3C 295 and 3C 207 is produced by this process. 


\section{Synchrotron radiation}

The strongest case for synchrotron $\mathrm{X}$-ray emission in an extragalactic jet is M87. In this jet, the X-ray spectra of all the individual knots are steeper than their radio or optical spectra (Marshall et al. 2002; Wilson \& Yang 2002), which argues against IC models. Further, knot HST-1 is highly variable in X-rays, strongly favoring synchrotron radiation (Harris, these proceedings; Harris et al. 2003). A detailed analysis of the Xray emission of the M87 jet is given by Perlman \& Wilson (these proceedings and in preparation). Harris \& Krawczynski (2002) present a list of other possible X-ray synchrotron sources.

\section{Problems}

\subsection{Why do some hot spots show SSC and others synchrotron $X$-rays?}

A promising suggestion has been made by Georganopoulos \& Kazanas (this conference). They propose that all hot spots are essentially the same, with the X-ray and optical synchrotron emission being significantly relativistically beamed along the source axis. The flow within the hot spots is relativistic and decelerating. The highest energy electrons (X-ray and optical emitting) are located in the immediate post (jet) shock region, and their beaming pattern is narrow. Further downstream, the

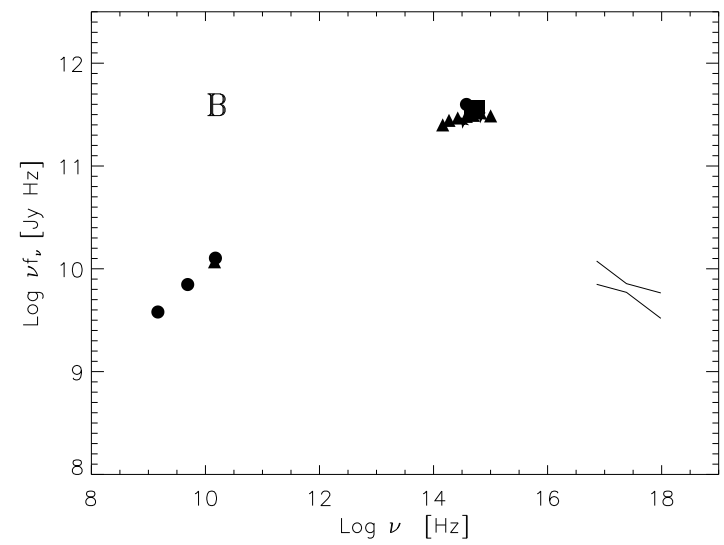

Fig. 2. Spectrum of knot B in the M87 jet (from Wilson \& Yang 2002)

flow is slower with a wider beaming pattern and these electrons emit the radio synchrotron and X-ray SSC emission. It will be important to confirm that the SSC emitting hot spots tend to be in objects with axis close to the plane of the sky and that the one sided, synchrotron emitting hot spots are viewed more pole-on.

\subsection{Synchrotron spectra}

X-ray synchrotron emitting electrons have energy half lives of order years and must be continuously reaccelerated. In some cases, the X-ray spectrum is not a simple extension of the radio-optical spectrum with a monotonically increasing spectral index with increasing frequency, as would be expected for synchrotron or IC losses. Examples of this unexpected behavior include the $\mathrm{W}$ hot spot in Pic A and knots A, B (Fig. 2) and possibly D in the M87 jet.

Wilson \& Yang (2002) discuss various explanations for this behavior, including the possibility that the $\mathrm{X}$ - 
rays come from a separate population of electrons from those responsible for the radio and optical emissions. One particular example of this type of model postulates that the volume within which the particles are accelerated is a function of the particle energy (cf. Perlman et al. 2001; Perlman \& Wilson in preparation). The spectrum observed at Earth would also be affected if the degree of beaming is a function of particle energy (cf. Georganopoulos \& Kazanas, these proceedings) and hence frequency. Lastly, Dermer \& Atoyan (2002) have shown that, when IC cooling on the CMB in the Thomson regime exceeds synchrotron cooling, a hardening in the electron spectrum is formed at electron energies where Klein-Nishina effects become important. This effect results from the reduction in Compton losses in the Klein-Nishina regime compared to the $\mathrm{dE} / \mathrm{dt} \propto \mathrm{E}^{2}$ losses of the Thomson regime. All of these processes may serve to modify the observed shape of the synchrotron spectrum.

This work was supported by NASA grants NAG 8-1027 and NAG 8-1755.

\section{References}

Begelman, M. C., Blandford, R. D. \& Rees, M. J. 1984, Rev. Mod. Phys., 56, 255

Brinkman, A. C. et al. 2002, A\&A, 396, 761

Brunetti, G., Setti, G. \& Comastri, A. 1997, A\&A, 325, 898

Brunetti, G. et al. 2001, A\&A, 372,755
Brunetti, G. et al. 2002, A\&A, 381, 795

Celotti, A., Ghisellini, G. \& Chiaberge, M. 2001, MNRAS, 321, L1 Dermer, C. D. 1995, ApJ, 446, L63 Dermer, C. D. \& Atoyan, A. M. 2002, ApJ, 568, L81

Garrington, S. T. et al. 1988, Nature, 331, 147

Hardcastle, M. J., Birkinshaw, M. \& Worrall, D. M. 2001, MNRAS, 323, L17

Harris, D. E., Carilli, C. L. \& Perley, R. A. 1994, Nature, 367, 713

Harris, D. E. et al. 2000, ApJ, 530, L81

Harris, D. E. \& Krawczynski, H. 2002, ApJ, 565, 244

Harris, D. E. et al. 2003, ApJ (submitted)

Kinkhabwala, A. et al. 2002, ApJ, 575, 732

Laing, R. A. 1988, Nature, 331, 149

Marshall, H. L. et al. 2002, ApJ, 564,683

Meisenheimer, K., Yates, M. G. \& Röser, H.-J. 1997, A\&A, 325, 57

Perlman, E. S. et al. 2001, ApJ, 551, 206

Schwartz, D. A. et al. 2000, ApJ, 540, 69

Schwartz, D. A. 2002, ApJ, 569, L23

Tavecchio, F. et al. 2000, ApJ, 544, L23

Wilson, A. S., Young, A. J. \& Shopbell, P. L. 2000, ApJ, 544, L27

Wilson, A. S., Young, A. J. \& Shopbell, P. L. 2001, ApJ, 547, 740 Wilson, A. S. \& Yang, Y. 2002, ApJ, 568, 133 